\title{
The presence of PI-rich vesicles is required for the PLC $\zeta$ activation according to mathematical modeling
}

\author{
by A.K. Tabachnik ${ }^{1 \#}$
}

1. Faculty of Physics, Lomonosov Moscow State University, 1/2 Leninskie gory, Moscow 119991, Russia

\# Correspondence: atabachnick@mail.ru

Received: 25.06.2021

Accepted: 29.06.2021

Published: 30.06 .2021

DOI: $10.52455 /$ sbpr.01.202102013

\section{Abstract}

Phospholipase C $\zeta$ (PLCZ) is an enzyme found in the cytoplasm and acrosome of mammalian spermatozoa. It catalyzes the reaction of phosphatidylinositol-4,5-phosphate hydrolysis into inositol-3-phosphate and diacylglycerol. PLCZ is present in the sperm cell acrosome and cytosol but doesn't significantly affect its metabolism. However, after the fusion of sperm and egg membranes, its activity increases as it begins to bind membranes of the egg. It is unknown why PLCZ is inactive in spermatozoa or any type of somatic cell.

A model describing the activity of PLC $\zeta$ in physiological calcium concentrations was developed. In this work, the modeling approach explains the absence of PLCZ activity in any type of mammalian cells but eggs. It was shown that the presence of phosphoinositide-rich vesicles is required for the PLC $\zeta$ activity in mature mammalian eggs.

Keywords: Phospholipase C ל, Oocyte, Spermatozoa, Calcium signalingt

\section{Introduction}

During mammalian fertilization, sperm induces a series of calcium oscillations in the egg that induce egg activation and early embryonic development [1]. Phospholipase C (PLC) $\zeta$ is a sperm-specific protein capable of causing $\mathrm{Ca}^{2+}$ release [2]. $\mathrm{PLC} \zeta$ is an enzyme that catalyzes the reaction of phosphatidylinositol-4,5-phosphate (PIP2) hydrolysis into inositol-3-phosphate (IP3) and diacylglycerol (DAG). It is present in the sperm cell acrosome and cytosol but doesn't significantly affect its metabolism. However, after the fusion of sperm and egg cell, its activity increases as PLC $\zeta$ begins to bind membranes of the egg cell and generate IP3 [3]. PLC $\zeta$ can also initiate calcium oscillations in oocytes without the sperm cell if its expression in the oocyte is artificially enabled [2]. Some eggs fertilized by PLCZ-null sperm can develop, albeit at significantly reduced efficiency, and after a significant time delay [4].
One of the main questions about PLC $\zeta$ is the reason for the absence of its activity in sperm cells, as well as in other cell types like Chinese hamster ovary cells (CHO) [5]. Several hypotheses are explaining the cause of exclusive PLC $\zeta$ activity in the oocyte (Fig. 1). One of them assumes that there is an unknown cofactor that activates PLC $\zeta$ (Fig. 1A). This cofactor is located in oocyte cytosol [3]. The alternative explanation suggests that in sperm PLC $\zeta$ is bound to some kind of inhibitor (Fig. 1B), and after fusion with the oocyte, the complex with inhibitor dissolves. This hypothesis partially contradicts data from [5], which demonstrated that when expressed in Chinese hamster ovary cells PLC $\zeta$ shows no significant impact on calcium concentration. None of these hypotheses are supported by direct evidence.

The alternative hypothesis is based on PIP2rich vesicles in oocytes (Fig. 1C, D). These vesicles have much higher relative surface space than the membrane, and it was demonstrated that a large part of PLC $\zeta$ bounds to these vesicles [6], [5].

The modeling approach has been successfully used to study the calcium activation mechanism in both the sperm and egg cells [7], [8], but no work exists on the PLC $\zeta$ activity regulation. In this work, we utilize the modeling approach to clarify the mechanisms regulating the PLC $\zeta$ activity in sperm and egg cells.

\section{Materials and methods}

\section{Computational model construction and integration}

To study the PLC $\zeta$ activity in mammalian eggs, a computer model was developed. First, the ordinary differential equations were constructed based on laws of chemical kinetics (either mass action law, Henry-Michaelis-Menthen kinetics, or Hill functions). Kinetic equation parameters were either taken from the literature or estimated based on existing experimental data. The final model consisted of 18 equations with two unknown parameters. The model was solved using the LSODA method. 

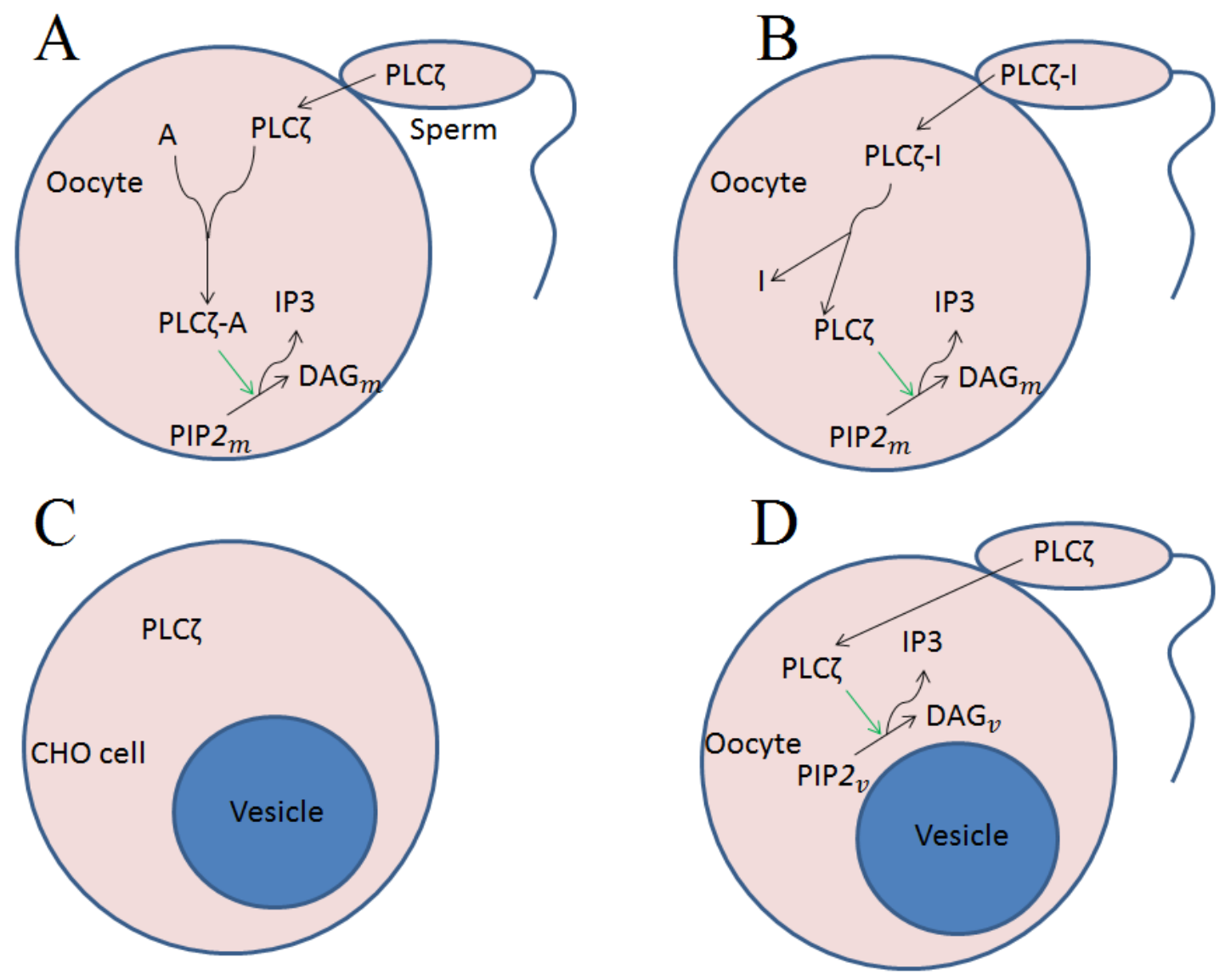

Figure 1. Major hypotheses explaining PLC $\zeta$ activity in the oocyte. (A) The first hypothesis is that the PLC $\zeta$ acquires the ability to cleave the oocyte membrane PIP2 (PIP2 $\left.{ }_{m}\right)$ and produce IP3 upon activation by an unspecified PLC $\zeta$ activator A present in oocytes. (B) The second hypothesis is the presence of an unspecified PLC $\zeta$ inhibitor in all cell types but eggs (C, D). The third hypothesis suggests that mammalian oocytes contain vesicles higher in PIP2 $\left(\boldsymbol{P I P 2 _ { v }}\right)$ content, and PLC $\zeta$ is targeted on the vesicle surface.

\section{Results and discussion}

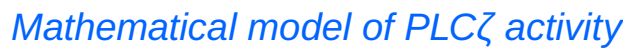

Before checking the hypotheses described above, an accurate mathematical model of PLCZ activity that can describe the existing experimental data [6],[9] was developed. According to multiple authors ([6],[9]) the unique feature of PLC $\zeta$ is its high cooperativity for $\mathrm{Ca}^{2+}$ binding, which is performed by its four EF-hand domains. Its Hill coefficient varies from 3.8 to 4.3 ([10], [11],[5],[9]) according to different sources. However, as it was shown below, such a high value for the Hill coefficient is incompatible with the data demonstrated in these articles. Therefore, the Hill coefficient was calculated using two different methods. It was shown that its value, according to the data from [10], is close to 1.1.

A simple mathematical model which could predict calcium dependence of $\mathrm{PLC} \zeta$ activity in physiologically relevant concentrations of calcium $\left(10^{-8}-10^{-6} \mathrm{M}\right)$ was created. It was based on Klotz equation (1), and a hypothesis that every following calcium ion binds with an equilibrium coefficient equal to the previous one multiplied on cooperativity constant $\alpha(2-5)$ :

$$
\begin{gathered}
B=\frac{\left[P L C z_{-} C a\right]+2 *\left[P L C z_{-} 2 C a\right]+3 *\left[P L C z_{-} 3 C a\right]+4 *\left[P L C z_{-} 4 C a\right]}{P L C t o t a l}= \\
=\frac{K e q *[C a]+2 * a * K e q^{2} *[C a]^{2}+3 * a^{3} * K e q^{3} *[C a]^{3}+4 * a^{6} * K e q^{4} *[C a]^{4}}{1+K e q *[C a]+a * K e q^{2} *[C a]^{2}+a^{3} * K e q^{3} *[C a]^{3}+a^{6} * K e q^{4} *[C a]^{4}}
\end{gathered}
$$

$$
\begin{aligned}
& K 2=K 1 * a \\
& K 3=K 1 * a^{2} \\
& K 4=K 1 * a^{3} \\
& K r=K 1 / K e q
\end{aligned}
$$

Here $\boldsymbol{K} \mathbf{1}$ is the constant for the first calcium ion binding, $\mathbf{K} \mathbf{2}$ - for the second calcium ion binding, K3 - for the third, $\boldsymbol{K} \mathbf{4}-$ for the fourth, $\boldsymbol{K e q}$ is the equilibrium constant for the first calcium ion binding reaction, $\boldsymbol{a}$ is cooperativity constant, $\left[\mathbf{P L C} \mathbf{z}_{-} \mathbf{C a}\right]$ is the concentration of $\mathrm{PLC} \zeta$ bound with one $\mathrm{Ca}^{2+}$ ion in the steady-state, $\left[P L C z \_2 C a\right]$ is the concentration of PLCZ bound with two $\mathrm{Ca}^{2+}$ ions in the steadystate, $\left[P L C z \_3 C a\right]$ with three, $\left[P L C z \_4 C a\right]$ with four. PLCtotal is the total concentration of all forms of PLCZ summarized. $\boldsymbol{B}$ is a parameter in the Klotz equation, representing the molar ratio of total PLCZ and calcium bound with it.

Using equations 2-5, a mathematical model based on mass action law was developed. Calcium concentration in this model was fixed, while variables represent dynamics of PLC $\zeta$ bound with different 
amount of calcium ions:

$$
\begin{gathered}
\frac{d[P L C z]}{d t}=-K 1 *[\mathrm{Ca}] *[\mathrm{PLC} z]+\mathrm{Kr} *\left[\mathrm{PLC} z_{-} \mathrm{Ca}\right] \\
\frac{d\left[\mathrm{PLC} z_{-} \mathrm{Ca}\right]}{d t}=K 1 *[\mathrm{Ca}] *[\mathrm{PLCz}]-\mathrm{Kr} *\left[\mathrm{PLC} z_{-} \mathrm{Ca}\right]- \\
-\mathrm{K} 2 *[\mathrm{Ca}] *\left[\mathrm{PLC} z_{-} \mathrm{Ca}\right]+\mathrm{Kr} *\left[\mathrm{PLC} z_{-} 2 \mathrm{Ca}\right] \\
\frac{d\left[\mathrm{PLC} z_{-} 2 \mathrm{Ca}\right]}{d t}=K 2 *[\mathrm{Ca}] *\left[\mathrm{PLC} z_{-} \mathrm{Ca}\right]-\mathrm{Kr} *\left[\mathrm{PLC} z_{-} 2 \mathrm{Ca}\right]- \\
-\mathrm{K} 3 *[\mathrm{Ca}] *\left[\mathrm{PLC} z_{-} 2 \mathrm{Ca}\right]+\mathrm{Kr} *\left[\mathrm{PLC} z_{-} 3 \mathrm{Ca}\right]
\end{gathered}
$$

$$
\begin{array}{rl}
\frac{d\left[P L C z_{-} 3 C a\right]}{d t}=K 3 & *[C a] *\left[P L C z_{-} 2 C a\right]-K r *\left[P L C z_{-} 3 C a\right]- \\
& -K 4 *[C a] *\left[P L C z_{-} 3 C a\right]+K r *\left[P L C z_{-} 4 C a\right]
\end{array}
$$

$\frac{d\left[P L C z_{-} 4 C a\right]}{d t}=K 4 *[C a] *\left[P L C z_{-} 3 C a\right]-K r *\left[P L C z_{-} 4 C a\right]$

Here, $\boldsymbol{K} \mathbf{1}$ is constant for the first calcium ion binding, $\boldsymbol{K} \mathbf{2}$ - for the second, $\boldsymbol{K} \mathbf{3}$ - for third, $\boldsymbol{K} \mathbf{4}$ for fourth, $K e q$ is the equilibrium constant for the first calcium ion binding reaction, $\boldsymbol{a}$ is cooperativity constant. The concentration of PLC,$[P L C z$ Ca] is the concentration of $\mathrm{PLC} \zeta$ bound with one $\mathrm{Ca}$ ion in the steady-state, $\left[P L C z \_2 C a\right]$ is the concentration of PLCZ bound with two Ca2+ ions in the steady-state, [PLCz_3Ca] - with three, $\left[P L C z \_4 C a\right]$ - with four.

We have investigated the steady-state of the model. The details are given in S1.

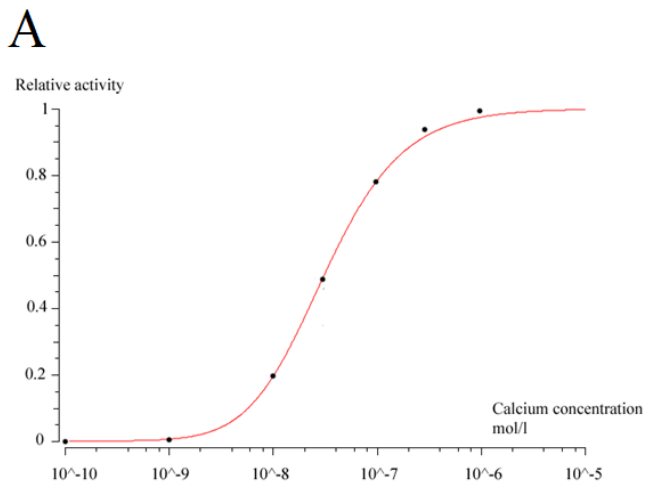

$\mathrm{C}$

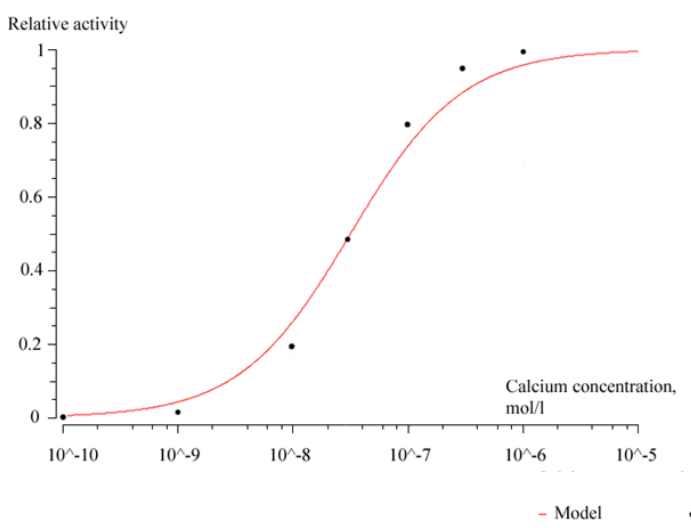

The model \% of activity was calculated as follows:

$\frac{0.03 *\left[P L C z_{-} 2 C a\right]+0.22 *\left[P L C z_{\_} 3 C a\right]+\left[P L C z_{-} 4 C a\right]}{\text { PLCtotal }}=\%$ of activity

This parameter depends on the concentrations of PLCZ forms associated with two, three or four calcium ions. The coefficients for recalculating the activity of the concentrations of these forms are taken from the data on the maximum activity of $\mathrm{PLC} \zeta$ with the deletion of one or two EF-hands [12]. For PLCZ bound to three calcium ions, the data on PLC $\zeta$ with one EF-hand deletion was used (in this case, activity was $22 \%$ of the native enzyme form activity). For $\mathrm{PLC} \zeta$ bound to two calcium ions, the data on PLCZ with two EF-hand deletion was used (in this case, activity was $3 \%$ of the native enzyme form activity).

Parameter optimization was performed using a genetic algorithm. The experimental enzyme activity in relation to the maximal (\% of activity) was obtained from the literature data.

The model predicted equilibrium constant $\mathbf{K e q}$ $=8.3^{*} 10^{8} M^{-1}$ and cooperativity constant $a=0.33$. Using these values, a dependency of PLC $\zeta$ activity on calcium concentration was built (Fig. 2A).

\section{Hill approximation of PLC $\zeta$ activity}

As an alternative approach to describing PLCZ activity, we used the Hill equation. The Hill equation for an enzyme has the following form:

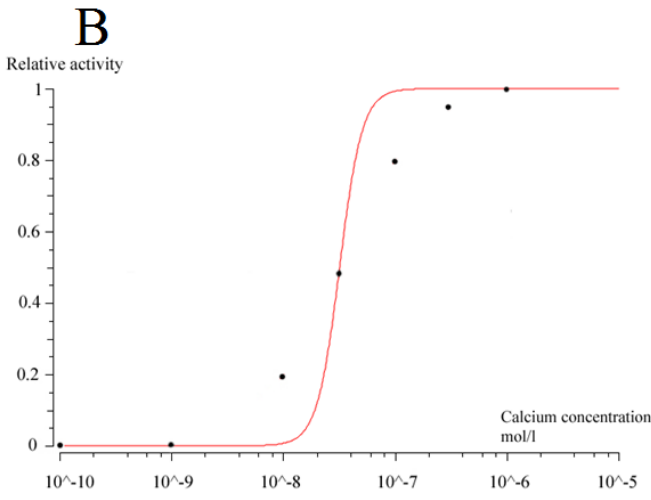

$\mathrm{D}$

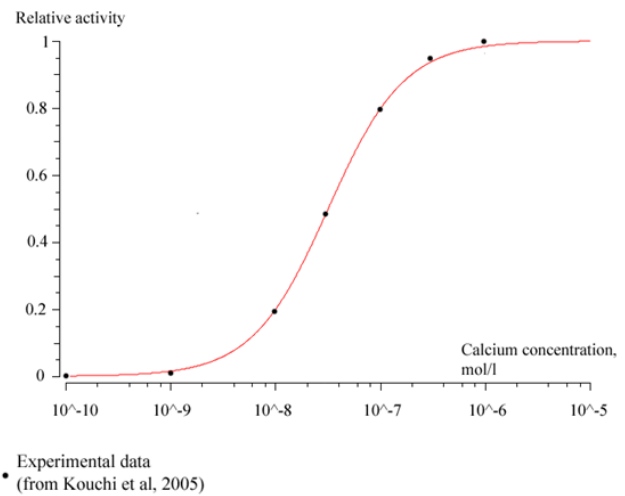

Figure 2. Validation of the models. (A) Validation of our calcium-binding model with experimental data from [10]. (B) Comparison of Hill equation for $n=4.3$ with experimental data from [10]. (C) Comparison of Hill equation for $n=0.9$ with experimental data from [10]. (D) Comparison of Hill equation for $n=1.1$ with experimental data from [10]. 


$$
\frac{E}{E m a x}=\frac{[C a]^{n}}{[E C 50]^{n}+[C a]^{n}}
$$

where $\boldsymbol{E}$ is PLCZ activity, $\boldsymbol{E} \max$ is on PLCZ maximum activity, EC50 is calcium concentration required for $50 \%$ activity, [Ca] is calcium concentration, $\boldsymbol{n}$ is Hill coefficient.

If we take the Hill coefficient from [11], $(n=4.3)$, we will receive calcium dependency, which can be observed on Fig. 2B. It approximates the experimental data with much higher standard deviation than our model described in (6)-(10). The same stands true for the Hill coefficient obtained from [12] $(n=0.9)$.

Hill coefficient could be estimated from data on calcium concentration at different activities (EC90 and EC10 - 90\% and 10\% of maximum activity accordingly).

$$
n=\frac{\log 10(91)}{\log 10(E C 90 / E C 10)}
$$

For PLCZ activity predicted by our model, EC90 is $2.7^{\star} 10^{-7} \mathrm{M}$, while EC10 is $5.3^{\star} 10^{-9} \mathrm{M}$. By using equation (13), we can find that Hill coefficient for PLC $\zeta$ is equal to 1.12 .

The same procedure was repeated for data from [11]. For PLCZ, EC90 is $4.3 \times 10^{-7} \mathrm{M}$, and EC10 is $8 \times 10^{-9} \mathrm{M}$. By using the same equation (13), we can find that the actual experimental Hill coefficient for PLCZ is approximately equal to 1.1 , which corresponds well with the predictions of our model.

The complete model of phospholipase $C$ activity in mammalian spermatozoa and egg cells

The complete model of PLC $\zeta$ activity on egg (Fig. 3A) and sperm membranes (Fig. 3B) was developed.
In the sperm and the egg, two similar reaction schemes were used. PLC $\zeta$ could bind calcium and the available plasmatic membranes independently, but only the form that was bound both to the membrane and four calcium ions could produce IP3. IP3 was removed from the system with a constant rate. The differences between two models were as follows: the egg contains vesicles rich in phosphoinositides, while sperm does not. All reactions occurring on the vesicle membrane duplicate the reactions occurring on the plasmalemma. The reaction constants between schemes did not differ, except for the presence of a separate constant for PLC binding to vesicles in the oocyte model and the rate constant for inositol-3phosphate degradation.

Two models were created using the schemes described above: a model for PLCZ activity in the oocyte (Fig.3, A) and sperm cell (Fig.3, B). The model equations are described in S2. The details of the model parameters are given in Table S1.

The dependence of IP3 concentration on PIP2 concentration on various membranes is shown in Fig. 4. A single steady-state existed in both of the models. The stationary concentration of IP3 in the spermatozoon was approximately 50-60 $\mathrm{nM}$, and in the oocyte, IP3 concentration was about $200 \mathrm{nM}$ (which is above the response threshold of $130 \mathrm{nM}$ [13] for IP3 receptors present both in sperm cells and oocytes [14], [15]). Thus, according to the point model, the activity of PLCZ alone should be sufficient to initiate calcium oscillations in the oocyte.

In the sperm cell, the final concentration of inositol-3-phosphate, depending on the concentration

A

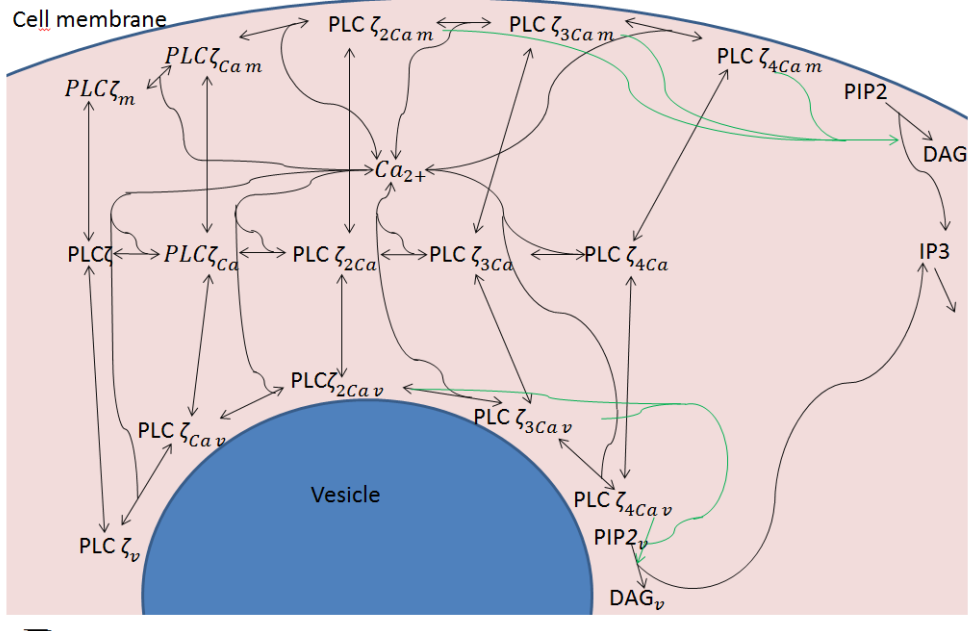

B

Figure 3. Scheme of the full model. (A) Reactions in the oocyte, $\mathrm{PLC \zeta}$ - calcium-free PLCZ, $\mathrm{PLC} \zeta_{\mathrm{Ca}}-\mathrm{PLC \zeta}$, bound with one calcium ion, $\mathrm{PLCl}_{2 \mathrm{Ca}}-\mathrm{PLC}$, bound with two calcium ions, $\mathrm{PLCZ}_{3 \mathrm{Ca}}$ - with three, $\mathrm{PLC \zeta}_{4 \mathrm{Ca}}-$ with four. PLC $\zeta_{m}-\mathrm{PLC \zeta}$, bound with cell membrane, $\mathrm{PLC \zeta}_{\mathrm{Cam}}-\mathrm{PLC \zeta}$, bound with the cell membrane and one calcium ion, $\mathrm{PLC \zeta}_{2 \mathrm{Cam}}-\mathrm{PLC \zeta}$, bound with the cell membrane and two calcium ions, $\mathrm{PLCl}_{3 \mathrm{cam}}$ - with the cell membrane and three calcium ions, $\mathrm{PLC}_{4 \mathrm{Cam}}$ - with the cell membrane and four calcium ions. PIP2 and PIP2 ${ }_{v}$ - are phosphatidylinositol-4,5 - bis-phosphates on cell and vesicle membrane accordingly. DAG and $D A G_{v}$ - diacylglycerol on cell membrane and vesicles accordingly. IP3 - inositol-3-phosphate. $\mathrm{PLCZ}_{v}$

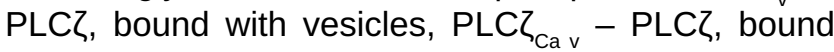
with vesicles and one calcium ion, $\mathrm{PLC}_{2 \mathrm{Cav}}-\mathrm{PLC}$, bound with vesicles and two calcium ions, $\mathrm{PLCl}_{3 \mathrm{Ca}}$ ${ }_{v}$ - with vesicles and three calcium ions, $\mathrm{PLC \zeta}_{4 \mathrm{Cav}}$ with vesicles and four calcium ions. (B) The sperm cell model is identical to the oocyte model except for the absence of the vesicles. 
A

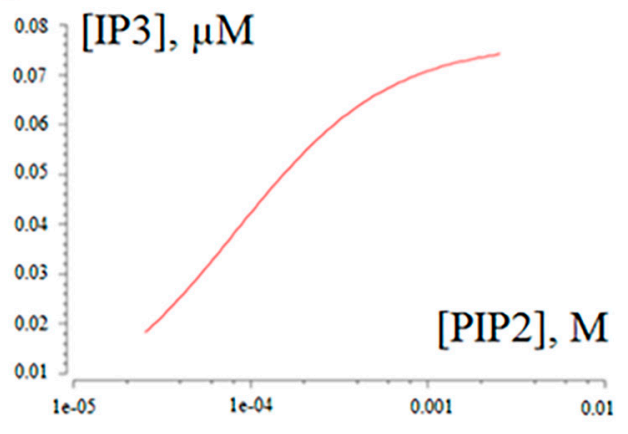

B

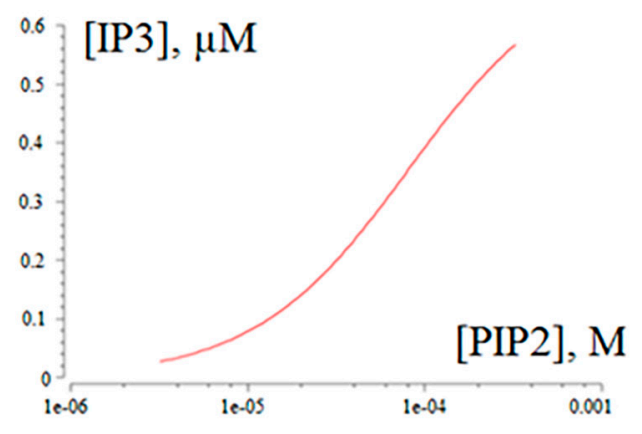

Figure 4. Results in the whole model. (A) Dependence of the IP3 concentration on sperm membrane PIP2 content. (B) Dependence of the IP3 concentration on PIP2 content in oocyte vesicles.

of PIP2, reaches a plateau long before reaching even half of the threshold concentration (130 nM).

A decrease in the concentration of phosphatidylinositol-4,5-bisphosphate on the surface of vesicles by a factor of 10 would reduce the final concentration of inositol-3-phosphate to approximately $20-30 \mathrm{nM}$ and eliminate the possibility of calcium oscillations in the oocyte solely due to the activity of PLCZ (Fig. 4B). This explains both the absence of oscillations upon initiation of PLCZ expression in somatic cells [2] and the absence of oscillations under similar conditions at the early stages of oocyte maturation, before the formation of phosphatidylinositol-4,5-bis-phosphate-rich vesicles [16].

\section{Conclusions}

Here we have built a mathematical model of PLC $\zeta$ activity in mammalian egg and sperm cells and validated it on existing experimental data. It was theoretically shown that the activity of PLC $\zeta$ in the sperm is insufficient for the synthesis of IP3 in sufficient quantities to initiate calcium oscillations. Its activity on vesicles in egg cells creates a total concentration of IP3 close to $200 \mathrm{nM}$, which is above the border value for initiation of Ca oscillations.

The results obtained support the hypothesis of the phosphoinositide composition of egg vesicles as the main factor in the effectiveness of PLC $\zeta$ as an initiator of calcium oscillations. They exclude the possibility of calcium oscillations in the sperm cell, which is consistent with all currently available experimental data.

\section{Author Contributions}

The whole work was completed by A.K.T.

\section{Acknowledgements}

Author is grateful to Miss Julia Jessica Korobkina and Dr. Anastasia Svesnikova (CTP PCP RAS, Moscow, Russia) for valuable discussions.

\section{References}

1. Saleh A, Kashir J, Thanassoulas A, SafiehGarabedian B, Lai FA, Nomikos M. Essential Role of Sperm-Specific PLC-Zeta in Egg Activation and Male Factor Infertility: An Update. Frontiers in Cell and Developmental Biology 2020;8. https://doi.org/10.3389/ fcell.2020.00028.

2. Yoshida N, Amanai M, Fukui T, Kajikawa $E$, Brahmajosyula $M$, Iwahori $A$, et al. Broad, ectopic expression of the sperm protein PLCZ1 induces parthenogenesis and ovarian tumours in mice. Development 2007;134:3941-52. https://doi.org/10.1242/ dev.007930.

3. Kashir J, Nomikos M, Lai FA. Phospholipase $C$ zeta and calcium oscillations at fertilisation: The evidence, applications, and further questions. Advances in Biological Regulation 2018;67:148-62. https://doi.org/10.1016/j. jbior.2017.10.012.

4. Hachem A, Godwin J, Ruas M, Lee HC, Buitrago MF, Ardestani G, et al. PLCZ is the physiological trigger of the $\mathrm{Ca} 2+$ oscillations that induce embryogenesis in mammals but offspring can be conceived in its absence. Development 2017. https://doi.org/10.1242/ dev.150227.

5. Phillips S V., Yu Y, Rossbach A, Nomikos M, Vassilakopoulou $V$, Livaniou E, et al. Divergent effect of mammalian $\mathrm{PLC} \zeta$ in generating $\mathrm{Ca} 2+$ oscillations in somatic cells compared with eggs. Biochemical Journal 2011;438:545-53. https://doi.org/10.1042/BJ20101581.

6. Nomikos M, Swann K, Lai FA. Starting a new life: Sperm PLC-zeta mobilizes the $\mathrm{Ca} 2+$ signal that induces egg activation and embryo development. BioEssays 2012;34:126-34. https://doi.org/10.1002/bies.201100127.

7. Atri A, Amundson J, Clapham D, Sneyd J. A single-pool model for intracellular calcium 
oscillations and waves in the Xenopus laevis oocyte. Biophysical Journal 1993;65:172739. https://doi.org/10.1016/S00063495(93)81191-3.

8. Simons J, Fauci L. A Model for the Acrosome Reaction in Mammalian Sperm. Bulletin of Mathematical Biology 2018;80:2481-501. https://doi.org/10.1007/s11538-018-0478-3.

9. Nomikos $M$, Theodoridou $M$, Elgmati $K$, Parthimos D, Calver BL, Buntwal L, et al. Human PLC exhibits superior fertilization potency over mouse PLC in triggering the $\mathrm{Ca} 2+$ oscillations required for mammalian oocyte activation. Molecular Human Reproduction 2014;20:489-98. https://doi. org/10.1093/molehr/gau011.

10. Kouchi Z, Fukami K, Shikano T, Oda S, Nakamura $Y$, Takenawa $T$, et al. Recombinant Phospholipase CZ Has High Ca2+ Sensitivity and Induces $\mathrm{Ca}+$ Oscillations in Mouse Eggs. Journal of Biological Chemistry 2004;279:10408-12. https://doi.org/10.1074/ jbc.M313801200.

11. Nomikos M, Blayney LM, Larman MG, Campbell K, Rossbach A, Saunders CM, et al. Role of Phospholipase C- $\zeta$ Domains in Ca2+-dependent Phosphatidylinositol 4,5-Bisphosphate Hydrolysis and Cytoplasmic Ca2+ Oscillations. Journal of Biological Chemistry 2005;280:31011-8. https://doi. org/10.1074/jbc.M500629200.

12. Kouchi Z, Shikano T, Nakamura Y, Shirakawa $\mathrm{H}$, Fukami K, Miyazaki S. The Role of EFhand Domains and C2 Domain in Regulation of Enzymatic Activity of Phospholipase Cद. Journal of Biological Chemistry 2005;280:21015-21. https://doi.org/10.1074/ jbc.M412123200.

13. De Young GW, Keizer J. A single-pool inositol 1,4,5-trisphosphate-receptor-based model for agonist-stimulated oscillations in $\mathrm{Ca} 2+$ concentration. Proceedings of the National Academy of Sciences 1992;89:9895-9. https://doi.org/10.1073/pnas.89.20.9895.

14. Kuroda Y, Kaneko S, Yoshimura Y, Nozawa S, Mikoshiba K. Are there inositol 1,4,5-triphosphate (IP3) receptors in human sperm? Life Sciences 1999;65:135-43. https:// doi.org/10.1016/S0024-3205(99)00230-1.

15. Cai X, Li X, Qi H, Wei F, Chen J, Shuai J. Comparison of gating dynamics of different IP $3 \mathrm{R}$ channels with immune algorithm searching for channel parameter distributions. Physical Biology 2016;13:056005. https://doi. org/10.1088/1478-3975/13/5/056005.

16. Parrington J, Swann K, Shevchenko VI, Sesay AK, Lai FA. Calcium oscillations in mammalian eggs triggered by a soluble sperm protein. Nature 1996;379:364-8. https://doi. org/10.1038/379364a0. 